Q. LI, K. ZHAO, A. PEURONEN, K. RISSANEN, D. ENDERS*, Y. TANG* (TSINGHUA UNIVERSITY, BEIJING AND SICHUAN UNIVERSITY, CHENGDU, P. R. OF CHINA; RWTH AACHEN UNIVERSITY, GERMANY; UNIVERSITY OF JYVÄSKYLÄ, FINLAND)

Enantioselective Total Syntheses of (+)-Hippolachnin A, (+)-Gracilioether A, (-)-Gracilioether E, and (-)-Gracilioether $\mathrm{F}$

J. Am. Chem. Soc. 2018, 140, 1937-1944.

\section{Syntheses of Plakortin Polyketides}

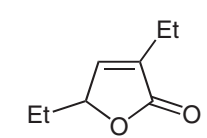

A

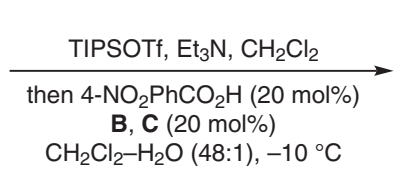

$68 \%, 91 \%$ ee

1. E, KHMDS

DME-HMPA (10:1)

$-78{ }^{\circ} \mathrm{C}$

2. DIBAL-H, $\mathrm{CH}_{2} \mathrm{Cl}_{2}$

D $\underset{\text { 3. } \mathrm{F}, \mathrm{PhMe}, \Delta}{\stackrel{-78}{\circ} \mathrm{C}}$

$72 \%, d r=1: 1$<smiles>CCCS(=O)(=O)c1nncn1-c1ccccc1</smiles><smiles>[CH]C1(CC(C=O)CC)C=C(CC)C(=O)O1</smiles>

D

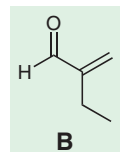

B

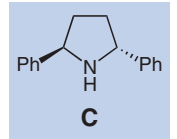

1. KHMDS, $\mathrm{PhSeCl}$ $\mathrm{THF},-78^{\circ} \mathrm{C}$

2. $\mathrm{MCPBA}, \mathrm{CH}_{2} \mathrm{Cl}_{2}$ $-78^{\circ} \mathrm{C}$

\section{3. $500 \mathrm{~W} \mathrm{Hg}$ lamp} acetone, $45^{\circ} \mathrm{C}$

\section{G} $\mathrm{CO}_{2} \mathrm{Me}$

$28 \%$

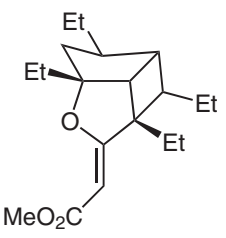

(+)-Hippolachnin A

\section{Category}

Synthesis of Natural

Products and

Potential Drugs

\section{Key words}

Plakortin

polyketides

(+)-hippolachnin A

gracilioethers

organocatalysis

[2+2]

photocycloaddition

HAT-triggered

oxygenation

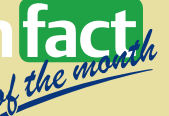

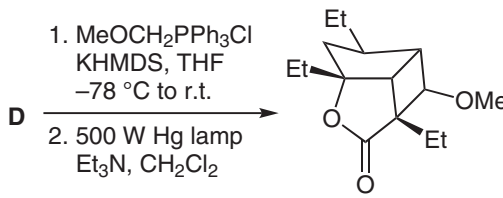

$58 \%$

1. I, n-BuLi

THF-HMPA (6:1)

$-78{ }^{\circ} \mathrm{C}$ to r.t.

2. $500 \mathrm{~W} \mathrm{Hg}$ lamp

D $\frac{\mathrm{CH}_{2} \mathrm{Cl}_{2}}{75 \%}$
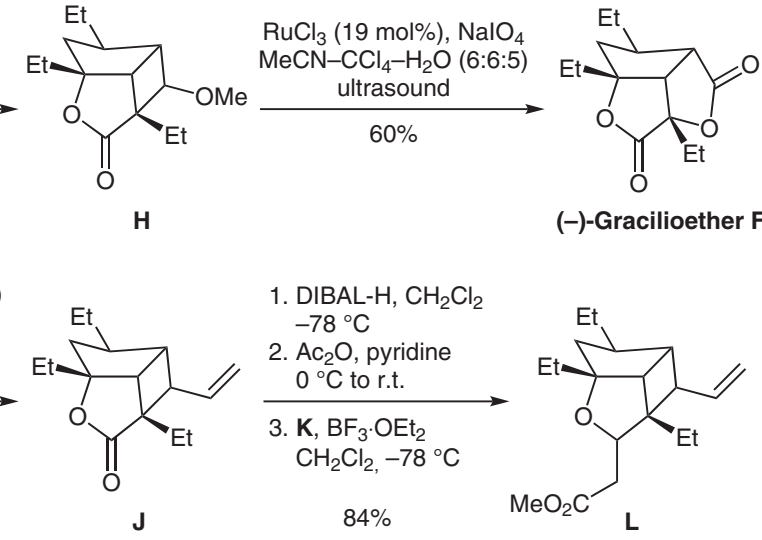

(-)-Gracilioether F
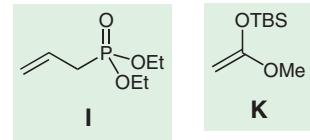

Significance: (+)-Hippolachnin A and the gracilioethers commonly feature a strained, bowl-shaped tricyclic core. Based on a [2+2]-photocycloaddition strategy, the authors report divergent syntheses of four different Plakortin natural products.
Comment: An organocatalytic, asymmetric 1,4addition afforded $\gamma$-butenolide $\mathbf{D}$, which served as a common intermediate. Chain elongation gave ester $\mathbf{G}$, which after desaturation and [2+2] photocycloaddition, yielded (+)-hippolachnin A.

SYNFACTS Contributors: Erick M. Carreira, Matthieu J. R. Richter

Synfacts 2018, 14(04), 0333 Published online: 16.03.2018

DOI: 10.1055/s-0037-1609334; Reg-No.: C01218SF 